Meta

Journal des traducteurs

Translators' Journal

\title{
La vie et les étranges aventures du texte Robinson
}

\section{Samia Barrada}

Volume 36, numéro 2-3, juin 1991

URI : https://id.erudit.org/iderudit/001978ar

DOI : https://doi.org/10.7202/001978ar

Aller au sommaire du numéro

Éditeur(s)

Les Presses de l'Université de Montréal

ISSN

0026-0452 (imprimé)

1492-1421 (numérique)

Découvrir la revue

Citer cet article

Barrada, S. (1991). La vie et les étranges aventures du texte Robinson. Meta, 36(2-3), 484-489. https://doi.org/10.7202/001978ar d'utilisation que vous pouvez consulter en ligne.

https://apropos.erudit.org/fr/usagers/politique-dutilisation/ 


\section{LA VIE ET LES ÉTRANGES AVENTURES DU TEXTE ROBINSON}

SAMIA BARRADA

École supérieure Roi Fahd de traduction, Tanger, Maroc

Après la Bible, le Robinson de Daniel Defoe est sans doute l'une des cuvres les plus traduites dans l'histoire de la littérature. Dans la bibliographie d'Ulrich Hermann Robinson und Robinsonaden 1 , on note que le roman de Defoe a été traduit en 27 langues.

Ulrich recense par ailleurs la longue lignée des avatars de Robinson - adaptations et imitations - au cours des XVIII e et XIX ${ }^{\mathrm{e}}$ siècle. En France, en Allemagne, en Autriche, en Finlande... chaque contrée avait «son» Robinson adapté à ses mœurs. Aujourd'hui encore la veine ne s'est pas tarie. Les Robinson du métro et Robinson des mers du Sud dans les collections verte ou rose voisinent sur les rayons des libraires avec d'autres titres plus incongrus tels Robinson-Cruauté ou La vie sexuelle de Crusoé.

Comment expliquer le formidable succès de cet ouvrage et le phénomène de réécriture en chaîne qu'il a enclenché? La lecture fastidieuse des trois tomes de l'œuvre de Defoe ne permet pas à elle seule d'élucider cette question. Le livre est mal écrit, le récit est encombré de réflexions moralisatrices qui décourageraient le lecteur le plus complaisant.

En fait, depuis sa parution en 1719, le texte Robinson a connu d'étranges aventures. Il a été questionné, corrigé, actualisé, reconstitué... Les adaptations, les imitations et les traductions témoignent de ce processus de révision. L'étude des traductions françaises permet d'entrevoir quelques-unes des transformations subies. Transformations qui expliquent en partie l'accession au statut de «classique» décerné à l'ouvrage.

Dès 1720 , la traduction de Thémisseul de Saint-Hyacinthe et Juste Van Effen ${ }^{2}$ paraît en France. Il faudra attendre 1835 pour que paraissent, presque simultanément, la traduction de Mme Amable Tastu ${ }^{3}$ (1re livraison, 12 mars 1835) et celle de Petrus Borel ${ }^{4}$ (21 mars 1835).

Les trois traductions ${ }^{5}$ présentent des variations importantes au niveau des choix d'écriture et au niveau thématique, chacune d'elle s'élaborant par rapport à une certaine conception de la traduction et du public qu'elle vise. Cette conception est lisible dans les préfaces, les notes, les remarques qui fournissent toutes sortes de justifications et de définitions de la traduction.

\section{LES PRÉFACES}

«Tradutore, tradittore», ce dicton italien qui scande l'histoire de la traduction, ramène toute tentative de définition de cette pratique à la question de la fidélité. Mais encore faut-il s'entendre sur ce que recouvre ce terme. Fidélité, certes? mais à qui ? à quoi? Les réponses divergent.

Pour les traducteurs de 1720 , il fallait que le roman de Defoe fût fidèle au bon goût français. Saint-Hyacinthe énumère toutes les belles infidélités qu'il a commises pour plaire à ses lecteurs :

Je ne m'étendrai pas beaucoup sur la traduction, elle n' est pas scrupuleusement littérale et l'on a fait de son mieux pour y aplanir un peu le style raboteux qui, dans l'original sent un peu trop le matelot, pour satisfaire à la délicatesse française. Cependant on n'a pas voulu le polir assez pour lui faire perdre son caractère essentiel qui doit être hors de la juridiction 
d'un traducteur fidèle. On a eu soin en récompense d'abréger les répétitions des mêmes pensées, de les déguiser par un changement de termes.

Cette profession de foi, qui ne peut qu'étonner le lecteur d'aujourd'hui, ne surprenait aucunement celui du XVIII ${ }^{e}$ siècle. Les principes énoncés par le Tourneur dans ses Nuits d'Young (Paris, 1769) sont adoptés par tous les traducteurs de l'époque. Le Tourneur montre comment il a tiré «de l'Young anglais un Young français qui put plaire à sa (ma) nation et qu'on put lire avec intérêt sans songer s'il est original ou copie».

De la même façon SH et VE ont su s'affranchir de leur modèle, pour répondre aux exigences du «bon goût» et de la «délicatesse française». «Traduire, écrivait Franz Rossenzweig, c'est servir deux maîtres, l'œuvre et le public». SH et VE ont élu délibérément le public.

Dans sa préface SH fait également allusion aux doutes qui subsistent quant au genre de l'œuvre. Était-ce une fiction ou une histoire vraie? Cette question qui était à l'ordre du jour dans les milieux littéraires anglais ne revêtait pas la même importance en France. Le roman n'y était pas considéré comme ce genre mensonger et méprisable auquel étaient assimilés les «romances» anglaises écrites principalement par des femmes telles Mrs Behn ou Mrs Manley, pour un public populaire avide d'histoires. SH cite les Aventures de Télémaque et montre que le problème de l'authenticité est un faux problème. Fable ou non qu'importe? Les Aventures de Télémaque sont fabuleuses aussi mais on n'en estime pas moins ce livre admirable. Tout comme l'ouvrage de Fénelon, Robinson Crusó́ 6 est investi d'une fonction didactique, et si l'histoire est véridique, ceci ne fait qu'appuyer la nécessité de corriger ce style «qui sent le matelot».

De son côté Mme Tastu, dans «l'avis de l'éditeur», critique les libertés de SH:

Saint Hyacinthe, tout en prétendant accommoder sa traduction aux délicatesses de notre langue, a tronqué ou défiguré les belles leçons de morale de Daniel Defoe (...) Mme Tastu a trop de goût pour avoir cherché à relever le style de Daniel Defoe. Elle ê̂t ainsi dénaturé son auteur dont une diction si naïve fait le principal mérite; mais elle a senti que cette nä̈veté même, comme celle de la Bible, avait sa noblesse, qu'elle pouvait dans son énergique franchise et sa grâce originale offrir aux jeunes les modèles aussi utiles que toutes les pompes du langage.

Pour T, la traduction doit être une fidèle réplique de l'original car toute transgression est perçue comme une altération de la réalité que le texte reflète. La traductrice, connue aussi comme auteure de livres pour enfants, ne manque pas, au passage, de souligner le caractère exemplaire du Robinson de Defoe. Toutefois, malgré ces principes de fidélité, la traduction de $\mathrm{T}$ reste attachée dans la pratique aux exigences stylistiques du XVIIIe. Quant à Pétrus Borel, après avoir dénoncé dans sa préface les erreurs commises par SH et VE, il s'en prend à la traduction de T qu'il juge médiocre car infidèle. Il présente la sienne comme la plus exacte.

Ainsi, de Saint Hyacinthe à Borel, la conception de la traduction a changé. Celle du XVIII siècle reste liée au purisme qui caractérise le siècle classique. Purisme qui faisait considérer toute traduction fidèle comme un péril pour l'équilibre de la langue française pure, stable, parfaite et donc hostile à tout apport étranger. Le XIX ${ }^{\mathrm{e}}$ siècle récuse cette attitude autarcique en reconnaissant à la fidélité au texte une place fondamentale.

\section{COMPARAISON DES TRADUCTIONS}

La comparaison de la traduction de $\mathrm{SH}-\mathrm{VE}$ avec celle de $\mathrm{T}$ réserve quelque surprise. À la lecture, on s'aperçoit en effet aisément que ces deux versions se «ressemblent». Certains passages sont quasiment identiques. Voici par exemple le passage où le père de Robinson tente de dissuader son fils de partir. 


\section{SH et VE}

Il me disait qu'il n'y avait que deux sortes de gens, les uns dénués de tout bien et sans ressources, les autres d'un rang supérieur et distingué, à qui il appartient de former des grandes entreprises et d'aller par le monde chercher les aventures afin de s'élever, et de se rendre fameux par une route peu frayée; que ce parti était au dessous de moi; que mon état était mitoyen, ou tel qu'on pourrait l'appeler le premier étage de la vie bourgeoisc...T
$\mathrm{T}$

Il me disait qu'il n'y avait que deux espèces de gens, les uns dénués de toute ressource, les autres d'un rang supérieur à qui il appartient de former de grandes entreprises et d'aller par le monde chercher les aventures parce que c'était là un moyen de s'élever, et de se rendre fameux par une route peu battue; qu'une telle carrière était (de beaucoup trop au-dessus ou trop) audessous de moi; que ma place était dans la classe moyenne celle qu'on peut appeler le premier étage de la vie bourgeoise...

Les seules modifications apportées par $\mathrm{T}$ sont la suppression de quelques ajouts et la substitution synonymique. De plus, certaines substitutions ou améliorations avaient déjà été opérées dans l'édition Panckoucke ${ }^{2}$. $\mathrm{T}$ reproduit aussi la ponctuation établie dans cette édition. Enfin, autre preuve, si besoin en est, du plagiat commis, T recopie servilement les erreurs de SH et VE. Ainsi l'omission du mot «aspiring» dans «aspiring superior fortunes» avait entraîné dans la première traduction un faux-sens. Au lieu de traduire par «les ambitieux» (cf. Borel), SH et VE et T écrivent «les autres d'un rang supérieur». Cette erreur s'étend à l'ensemble de la phrase à la recherche d'une cohérence. «Who went abroad upon adventures to rise by enterprise» devient «à qui il appartient de former de grandes entreprises et d'aller chercher par le monde des aventures afin de s'élever...»

Plus d'un siècle s'est écoulé avant de voir paraître une nouvelle traduction de Robinson Crusoé et celle qui finalement a été entreprise est d'une fidélité surprenante à l'ancienne!

Comment expliquer que Mme Tastu n'ait pas respecté les principes prônés dans sa préface? La réponse à cette question semble se situer dans le succès même de la première traduction. Non seulement de nombreuses adaptations ont été réalisées à partir du texte de $\mathrm{SH}$ et VE mais, chose plus remarquable, d'autres traductions. Ferdinand Brunot nous apprend, en effet, que la traduction allemande du roman de Defoe a été effectuée à partir de la version française.

Un critique disait en 1775 à propos de la première adaptation de la traduction de SH et VE:

L'on ne doute pas qu'elle ne devienne en quelque sorte un classique, d'autant plus qu'on vient de l'adopter dans plusieurs collèges.

Ce statut de «classique» a également été décerné à la traduction de 1720 par Paul Dottin, un critique du $\mathrm{XX}^{\mathrm{e}}$ siècle, qui estime :

Elle est restée la meilleure traduction de Robinson Crusoé, elle rend en français du $18^{e}$ siècle un roman écrit en anglais du $18^{e}$ siècle. Certes, elle n'est pas parfaite, car au lieu d'évoquer les sombres logis puritains de la cité ou de l'East-End elle dégage un parfum de boudoirs et des salons légers et spirituels. Mais elle est parfaitement adaptée au génie français et plaît comme une auvre originale; à beaucoup d'égards, elle mériterait de devenir un live classique.

Malgré toutes les erreurs et infidélités qu'on lui a reprochées - et qui ont été rectifiées en partie par les éditeurs - la traduction de SH et VE a longtemps été jugée la 
meilleure. Euvre originale à part entière, elle est appréciée pour ses qualités littéraires et introduites dans le domaine des auvres consacrées : les classiques. Lorsque Mme Tastu imite Saint Hyacinthe et Van Effen c'est avec la même déférence que lorsqu'on imitait autrefois les auteurs grecs et latins. Pour les célébrer. En revanche, Borel, en simple médiateur, s'efforce de rendre scrupuleusement l'original.

\section{Texte original}

He told me it was for men of desperate fortunes on one hand or aspiring superior fortunes on the other who went abroad upon adventures, to rise by enterprise and make themselves famous in undertakings out of nature out of the common road; that these things were all either too far above me, or too far below me; that mine was the middle state, or might be called the upper stations of low life...

\section{Borel}

Il n'y a que les hommes dans l'adversité ou les ambitieux qui s'en vont chercher aventures dans les pays étrangers pour $s$ 'élever par entreprise et se rendre fameux par des actes en dehors de la voie commune. Ces choses sont de beaucoup trop au-dessus ou trop au-dessous de toi; ton état est le médiocre ou ce qui peut être appelé la première condition du bas étage...

Les seules améliorations pratiqués par B sont la ponctuation et le passage du style indirect au style direct. Notons toutefois que cette dernière transformation avait déjà été effectuée dans l'Édition Eymery (1813) de Robinson Crusoé destinée aux enfants, dans le souci d'animer le récit. Le dialogue est depuis longtemps connu pour ses vertus pédagogiques et de nombreuses adaptations se présentent sous la forme de «Robinson Crusoé» raconté par un père à ses enfants. Borel n'oublie pas de signaler son infidélité en note.

\section{LES BELLES INFIDÈLES}

Le style de Defoe semble avoir posé de sérieux problèmes aux traducteurs. La clarté et la brièveté exigées par les littéraires s'accordent mal avec la fidélité due au texte anglais.

Alors que $B$ respecte scrupuleusement le texte anglais, reproduisant les répétitions, les digressions, les complétives, caractéristiques du style de Defoe, très proche du discours oral, SH et VE épurent le texte, le polissent, gommant tout ce qui «sent le matelot». Nombreux sont les passages qui témoignent de cette volonté de stylisation.

Après la première tempête de Robinson, un marin l'interpelle: «But you're but a fresh water sailor Bob, come let us make a bowl of punch and we'll forget all that».

$B$ traduit «tu n'est qu'un marin d'eau douce, Bob, viens que nous fassions un bol de punch et que nous oublions tout cela».

SH et VE, imités par T, rehaussent cette exclamation par une périphrase précieuse :

Vous n'êtes qu'un novice, mettons-nous à faire du punch et que les plaisirs de Bacchus nous fassent entièrement oublier la mauvaise humeur de Neptune.

Il arrive que $\mathrm{T}$ conteste le lyrisme des traducteurs de 1720 . On remarque d'ailleurs qu'au-delà des cent premières pages, sa traduction s'éloigne de celle de $\mathrm{SH}$ et VE, et devient plus exacte.

\section{Ex.: For sudden joys like griefs confound at first}

La surprise qui naît de joie ou de douleur suspend les fonctions de l'esprit et du cœur (SH et VE).

Le premier effet des joies comme des peines soudaines est une violente commotion $(T)$.

Car le premier effet des joies et afflictions soudaines est d'anéantir (B). 
La publication de la traduction de $\mathrm{B}$ a eu, sans nul doute, quelque incidence sur la traduction de $\mathrm{T}$ en engageant le débat sur la fidélité. Une véritable querelle ne tarda pas à s'engager entre les deux traducteurs.

$T$ se défend en invoquant la «basse jalousie» et les «insinuations» de certains mais c'est surtout au fil des pages, dans les notes qui jalonnent les deux textes que les traductions se trouvent confrontées.

$B$ signale les fautes de $\mathrm{T}$ : fautes de français, faux sens, contresens, omissions, amplifications.

$\mathrm{T}$, dans la $2^{\mathrm{e}}$ édition de sa traduction, répondra en signalant, à son tour, les erreurs du «traducteur fidèle» comme elle le nomme, non sans ironie. Les erreurs qu'elle souligne ne nécessitent d'ailleurs pas toujours de correction. En revanche les remarques de $\mathrm{B}$ sont le plus souvent fondées. T en prend note dans sa $2^{\mathrm{e}}$ édition.

\section{LÀ OŨ LA TRADUCTION DEVIENT CENSURE}

De nombreux passages du roman de Defoe portent atteinte à la religion catholique. Aussi le livre a-t-il souvent été jugé dangereux. On l'interdit en Espagne en 1756 à cause des attaques contre l'Inquisition. Il fallut attendre 1826 puis 1835 pour que paraissent à Paris les premières traductions (abrégées) en espagnol.

Dans sa préface, SH fait état de cet aspect du roman mais pour prévenir toute inquiétude en assurant l'innocuité de l'œuvre de Defoe. «Il ne se peut rien trouver de plus orthodoxe que le pauvre Robinson Crusoé, rien n'est plus édifiant que les réflexions continuelles qu'il fait pour justifier la providence divine.»

Toutefois, certains passages ont dû sembler bien dangereux si l'on juge la manière avec laquelle nos traducteurs les ont abordés.

Texte anglais :

I had once a Mind to have gone to the Brasils, and have settled myself there; for I was, as it were, naturaliz'd to the place; but I had some little scruple in my Mind about religion, which insensibly drew me back, of which I shall say more presently. However, it was not Religion that kept me from going there for the present; and as I had made scruple of being openly of the Religion of the country all the while I was among them having of late thought more of it (than formerly) when I began to think of living and dying among them, I began to regret my having profess'd myself a Papist, and thought it must not be the best Religion to die with.

J'avais assez d'envie quelquefois de m'établir entièrement dans le Brésil où j'étais comme naturalisé, mais j'étais retenu par quelques scrupules de conscience. Il est bien vrai qu'autrefois j'avais eu assez peu de délicatesse pour professer extérieurement la religion dominante du pays, et que je ne voyais pas encore qu'il y eut là un si grand crime; mais néanmoins, en y pensent plus mûrement je jugeais qu'il n'était pas sûr pour moi de mourir dans une pareille dissimulation et je me repentais d'en avoir jamais été capable (SH et VE).

Il me vint une fois envie de partir et de m'établir tout à fait au Brésil, car j'y étais pour ainsi dire naturalisé; mais quelques petits scrupules religieux s'élevèrent dans mon esprit et me détournèrent insensiblement de ce projet. Ce n'était pourtant pas tout à fait la religion qui m'arrêtait alors, et comme je ne m'étais jadis fait aucun scrupule de professer ouvertement le culte du pays tout le temps que j'y avais demeuré, il n'est pas dit que je ne l'eusse pas fait encore. Seulement comme j'avais depuis peu réfléchi sur ce sujet plus que je n'avais fait auparavant, quand je venais à penser qu'il s'agissait d'aller vivre et mourir en pareille compagnie, je commençais à me repentir d'avoir professé le catholicisme, et à croire que ce pouvait bien ne pas être la meilleure religion pour y mourir. (T)

Tastu traduit la totalité de l'énoncé sans rien omettre. Cette fidélité est cependant excusée par une note en bas de page, rappelant que Robinson avait été élevé dans la religion protestante. Saint-Hyacinthe et Van Effen remanièrent complètement le texte et 
lui donne une tout autre signification. Ici, Robinson a un problème de conscience, il se repent d'avoir simulé la conversion et refuse de mourir dans le mensonge.

Quant à Borel, il choisit tout simplement la censure: la dernière phrase où Robinson refuse de mourir en catholique est supprimée. Et la traduction de Borel continue d'être éditée avec ce coup de ciseau (cf. Gallimard, Bibliothèque de la Pléiade, p. 28). Pour tous les autres passages où sont développées les idées de Robinson sur la religion, Borel censure, SH et VE tentent d'atténuer les atteintes au catholicisme, Tastu s'efforce de respecter le texte mais coupe dès que les attaques deviennent trop insistantes.

De toute évidence, il était difficile pour les traducteurs des XVIII ${ }^{\mathrm{e}}$ et $\mathrm{XIX} \mathrm{X}^{\mathrm{e}}$ siècles de concilier fidélité à l'original et respect des principes religieux du lecteur. Les éditeurs auront encore moins de scrupules et opteront systématiquement pour la censure.

Nous venons de voir comment les traductions ont contribué à hausser le roman de Defoe au niveau d'un classique, dans le sens d'un «stéréotype de la culture littéraire ${ }^{7}$ ».

Corrigé, embelli, remodelé, épuré... l'ouvrage de Defoe, présenté à sa parution comme un récit de voyage «authentique», sera transformé en une œuvre littéraire, écrite et conforme aux règles d'un genre encore en gestation: le roman. Un genre qui, tel notre écumeur d'épaves, s'est construit «de toutes pièces» contre les «romances» qui dépravaient les lecteurs et à partir des biographies spirituelles. Rappelons, en effet, que pour les premiers lecteurs de Defoe, Robinson était lu comme un ouvre allégorique : l'histoire se noue dans le dénuement et le péché et se dénoue dans la richesse et la rédemption. Mais nous avons vu comment certaines réflexions religieuses de notre solitaire ont été censurées par les traducteurs. Les méditations ainsi que les citations bibliques qui informent l'aventure robinsonnienne en lui conférant une valeur symbolique finiront par disparaitre totalement. Les traducteurs mais aussi les éditeurs, les adaptateurs et autres imitateurs «laïciseront» la robinsonnade et orienteront résolument l'œuvre vers un public de jeunes. Le récit de Defoe sera soumis à toutes sortes de transformations: narratives, stylistiques, thématiques,... déterminées et justifiées par une certaine image de l'enfance. Métamorphosé en un texte pédagogique, il sera durant tous les $\mathrm{XIX}^{\mathrm{e}}$ et $\mathrm{XX}^{\mathrm{e}}$ siècles considéré comme un texte modèle susceptible d'être lu par les jeunes et enseigné dans les classes.

\section{NOTES}

1. Hermann Ullrich (1898): Robinson und Robinsonnadon. Bibliographie gestrichte Kritik, Weimar, in $8^{\circ}$ $\mathrm{XXIX}+248 \mathrm{p}$.

2. Daniel Defoe (1721): La Vie et les aventures surprenantes de Robinson Crusoé, trad. par Thémiseul de Saint-Hyacinthe et Juste Van Effen. Amsterdam, l'Honoré et Châtelain, 6 tomes en 3 vol., in 12.

La Vie est les aventures de Robinson Crusoé, ancienne trad. rév. corr. sur la belle édition donnée par Stockdale en 1790, augm. de la vie de l'auteur... Paris, Ve Panckoucke, an VIII, (Trad. de Saint-Hyacinthe et J. Van Effen).

3. Aventures de Robinson Crusoé, trad. par Mme A. Tastu, suivies d'une notice sur Foé et sur le matelot Selkirk par Denis Reybaud... Paris, Moutardier, 1835, 2 tomes.

4. Robinson Crusoé, trad. de Petrus Borel, enrichie de la vie de D. Defoe par Philarète Chasles, d'une notice sur le matelot Selkirk. Paris, F. Borel et A. Varenne, 1836, 2 tomes en 1 vol. in 80.

5. Nous désignerons les traductions par les abréviations suivantes:

SH et VE: traduction de Saint Hyacinthe et Van Effen

$\mathrm{T}$ : traduction de Mme Tastu

$\mathrm{B}$ : traduction de Borel

6. Du point de vue de l'éthique classique, le roman a une fonction instructive, il doit édifier ses lecteurs, leur servir d'exemple, leur montrer le chemin, les erreurs à éviter. Les romanciers du $19^{\circ}$ siècle, conscients de la mission qui leur est assigné, ne cesseront de la rappeler dans leurs préfaces. Cf. Anthologie des préfaces des romans français du XIXe siècle, Julliard, 1964.

7. Robert Escarpit (1970): Le littéraire et le social, Paris, Flammarion, coll. Champs, $312 \mathrm{p}$. 